\title{
Life Prediction on a T700 Carbon Fiber Reinforced Cylinder with Limited Accelerated Life Testing Data
}

\author{
Ma Xiaobing ${ }^{1}$ and Zhang Yongbo ${ }^{2}$ \\ ${ }^{1}$ School of Reliability and Systems Engineering, Beihang University, Beijing 100191, China \\ ${ }^{2}$ Research Center of Small Sample Technology, Beihang University, Beijing 100191, China \\ Correspondence should be addressed to Zhang Yongbo; zhang19840504@163.com
}

Received 14 July 2014; Revised 10 September 2014; Accepted 10 September 2014

Academic Editor: Shaofan Li

Copyright (c) 2015 M. Xiaobing and Z. Yongbo. This is an open access article distributed under the Creative Commons Attribution License, which permits unrestricted use, distribution, and reproduction in any medium, provided the original work is properly cited.

\begin{abstract}
An accelerated life testing investigation was conducted on a composite cylinder that consists of aluminum alloy and T700 carbon fiber. The ultimate failure stress predictions of cylinders were obtained by the mixing rule and verified by the blasting static pressure method. Based on the stress prediction of cylinder under working conditions, the constant stress accelerated life test of the cylinder was designed. However, the failure data cannot be sufficiently obtained by the accelerated life test due to the time limitation. Therefore, most of the data presented to be high censored in high stress level and zero-failure data in low stress level. When using the traditional method for rupture life prediction, the results showed to be of lower confidence. In this study, the consistency of failure mechanism for carbon fiber and cylinder was analyzed firstly. According to the analysis result, the statistical test information of carbon fiber could be utilized for the accelerated model constitution. Then, rupture life prediction method for cylinder was proposed based on the accelerated life test data and carbon fiber test data. In this way, the life prediction accuracy of cylinder could be improved obviously, and the results showed that the accuracy of this method increased by $35 \%$.
\end{abstract}

\section{Introduction}

In recent years, T700 carbon fiber replacing T300 becomes the new general carbon fiber since densification gives the T700 higher tensile strength [1-6]. The cylinder of new special equipment consists of T700 carbon fiber composite materials, glass fiber composites, and aluminum alloy. The main role of aluminum alloy is to improve the axial modulus and corrosion resistance of cylinder, the glass fiber composite material is to guarantee the aluminum alloy being adapted to higher working because of large prestress, and the carbon fiber composite material is to improve the strength and modulus of the cylinder and it is also the main load-bearing material between the three-layer materials.

The introduction of carbon fiber into the new generation cylinder improves the failure stress of the cylinder, but it also brings new problem to the reliability evaluation of the cylinder. The cylinder wound by T700 carbon fiber exhibits long life and high reliability in high-speed rotation mode, and the sufficient data cannot be obtained by the traditional life tests. Therefore, the accelerated test must be introduced, and the reliability index in the normal stress could be extrapolated by the statistical analysis using the high stress level data [7]. The maximum likelihood estimation method $[8,9]$ is very suitable for the censored data analysis, but this method only has good properties for the large sample. And it needs to iterate for the transcendental equations; sometimes the computation is difficulties and not convergence. The integral best linear unbiased estimation method $[10,11]$ solves problems by regression analysis based on linear transformation acceleration model. The information between different stresses is used comprehensively, and the estimation accuracy can be improved. But the evaluation accuracy is still unable to meet the actual engineering requirement of the cylinder structure with great life dispersion.

This study found that the failure mode of the cylinder wound by T700 carbon fiber was carbon fiber breakage in the working mode with high-speed rotation. Therefore, there would be some relationship between the rupture life of fiber carbon and the cylinder. In this study, we analyzed the 
mechanical properties of the cylinder composite material and obtained the ultimate failure stress by mixing rules, which were verified by the static pressure burst test method. Then we designed accelerated life tests on the carbon fiber and the cylinder. According to the test results, we proposed the integral best unbiased estimate method for the cylinder life prediction. The evaluation accuracy was greatly improved by comprehensive evaluation using the information of T700 carbon fiber based on the test results of the accelerated equivalence and failure mechanisms.

\section{Mechanical Analysis}

2.1. Mechanical Analysis of the Composite Material. The new generation cylinder consists of aluminium alloy, glass fiber, and T700 carbon fiber, and its structure is illustrated in Figure 1.

The main role of the aluminium alloy is to improve the axial modulus and anticorrosion of the cylinder. The glass fiber is to impose prestress to the aluminium alloy according to winding, and so it could improve the maximum capacity. The carbon fiber is the main bearing materials and for their high strength and modulus the ultimate failure stress of cylinder is greatly improved. The mechanical properties of the glass fiber and the carbon fiber can be obtained by mixing rules according to tensile strength, modulus, and the volume content of each fiber. Thus, the overall mechanical properties of the cylinder could also be estimated by the mixing rule basing on the material properties and structures.

According to the property test of T700 carbon fiber, the tensile strength is $4900 \mathrm{Mpa}$, the modulus is $230 \mathrm{GPa}$, and the strain is approximately $2 \%$. Also, the matrix strength of the composite material is $85 \mathrm{MPa}$, the modulus is $2.8 \mathrm{Gpa}$, and the strain is $4 \%$. The mix rule is

$$
X_{t}=\sigma_{f} V_{f}+\sigma_{m}\left(1-V_{f}\right) \text {, }
$$

where the $X_{t}$ is tensile strength of composite, $\sigma_{f}$ is the tensile strength of fiber, $\sigma_{m}$ is the tensile strength of matrix, and $V_{f}$ is the fiber volume. Therefore, for the fiber volume of composite material used in this study is $72 \%$, the tensile strength of this composite would be calculated as $3551.8 \mathrm{MPa}$.

For the glass fiber, the tensile strength is $3400 \mathrm{MPa}$, the modulus is $91 \mathrm{GPa}$, and the strain is approximately $3.7 \%$. And the matrix is the same with the T700 carbon fiber reinforced composite. While the percentage of fiber volume is $76 \%$, according to the mix rule, the tensile strength of this glass fiber reinforced composite would be calculated as 2604.4.

The lining of the cylinder is aluminium alloy, its elastic modulus is $70 \mathrm{GPa}$, yield strength is $610 \mathrm{Mpa}$, tensile strength is $640 \mathrm{Mpa}$, and strain is greater than $4 \%$ [12].

2.2. Failure Stress Analysis of the Cylinder. The tensile modulus of the glass fiber and the aluminium alloy is significantly lower than the outer carbon fiber composite material by the previous analysis. And according to the thickness of each laminate listed in Table 1, it is obvious that the carbon fiber is the main load-bearing material in the entire cylinder. When the outer T700 carbon fiber reinforced composite

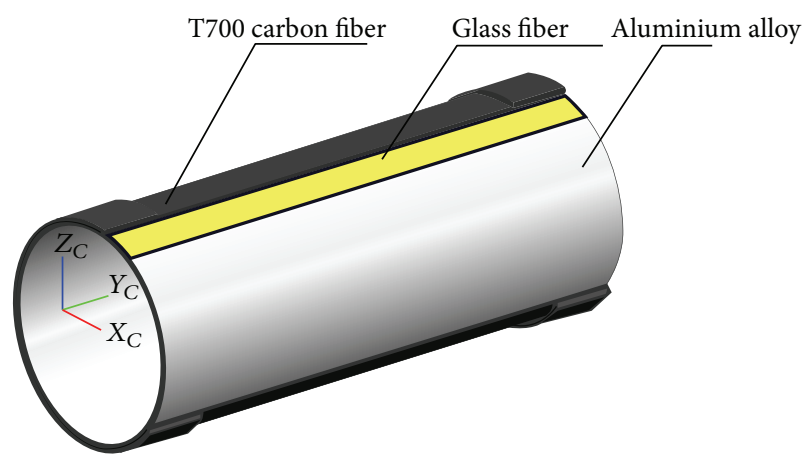

Figure 1: Structure of the cylinder.

is destructed, the glass fiber and aluminum alloy would be instantaneously destroyed due to the large load. The stress-strain curves of glass fiber and carbon fiber reinforced composite materials presented to be linear like the tensile stress-strain curves of glass fiber and carbon fiber that are shown in Figures 2 and 3.

The bearing stress of glass fiber is $380 \mathrm{Mpa}$ that is the reverse stress to impose on the aluminum alloy; therefore, its bearing stress is $1777 \mathrm{Mpa}$ when the conditions are the same with the outer carbon fiber. The tensile strength of aluminum alloy is $640 \mathrm{MPa}$, we consider that it imposed the reverse prestress of $380 \mathrm{MPa}$, its bearing stress is $260 \mathrm{Mpa}$ when the breaking elongation is $2 \%$, and its thickness is $1.2 \mathrm{~mm}$. The thickness of glass fiber and the carbon fiber is $0.85 \mathrm{~mm}$ and $1.5 \mathrm{~mm}$, respectively. Therefore, according to the mixing rule, the ultimate failure stress of the cylinder is theoretical as

$$
\sigma=\frac{\sigma_{l} \times t_{l}+\sigma_{g} \times t_{g}+\sigma_{f} \times t_{f}}{t_{l}+t_{g}+t_{f}}=2004 \mathrm{MPa}
$$

The strip tensile test method [13] for ultimate failure stress of the cylinder cannot meet the requirements of test accuracy with a few samples. The NOL [13] ring stretching method is sensitive to the boundary effects and sample processing is very difficult. Therefore, we chose the blasting static pressure method [14] for the ultimate failure stress testing which dealt with the data as a whole and thus could reflect the mechanical properties better.

The test samples were produced according to the national standard GB/T15560, and the processing technical, raw materials were the same with the cylinder. Figure 4 shows the size of this test sample. As shown, two ends of the cylinder were strengthened by carbon fiber layers of $30 \mathrm{~mm}$ width. Table 2 shows the parameters of this static pressure burst test. Table 3 shows the test result.

It can be seen from Table 3 that the mean of the ultimate test failure stress is $2067.6 \mathrm{MPa}$, which is slightly higher than the theoretical prediction of $2004 \mathrm{Mpa}$. It may be the reason that the overall performance of the cylinder material would be slightly higher than fiber samples. The parameters used for theoretical prediction were the mean value of the tensile test results of fiber sample, and thus its volatility is relatively large. Another main reason may be that the length of the test sample in the static pressure burst test was required to be 
TABLE 1: Geometry parameters and properties of each laminate.

\begin{tabular}{lccc}
\hline & Aluminium alloy & Glass fiber & Carbon fiber \\
\hline Thickness $(\mathrm{mm})$ & 1.20 & 0.85 & 1.50 \\
Modulus $(\mathrm{GPa})$ & 71 & $70,20\left(90^{\circ}\right)$ & $163,8\left(90^{\circ}\right)$ \\
Density $\left(\mathrm{g} / \mathrm{cm}^{3}\right)$ & 2.8 & 2.15 & 1.58 \\
\hline
\end{tabular}

TABLE 2: Parameters of the static pressure burst test.

\begin{tabular}{|c|c|c|c|c|}
\hline \multirow{2}{*}{$\begin{array}{l}\text { Aluminium alloy } \\
(\mathrm{mm})\end{array}$} & \multicolumn{2}{|c|}{ Process conditions } & \multirow{2}{*}{ Resin formula } & \multirow{2}{*}{ Curing system } \\
\hline & Glass fiber & Carbon fiber & & \\
\hline $\begin{array}{l}\text { Thickness: } 1.2 \\
\text { Length: } 350\end{array}$ & 5 layers & 10 layers & Proprietary formula & $75^{\circ} \mathrm{C} / 4 \mathrm{~h}+80^{\circ} \mathrm{C} / 12 \mathrm{~h}$ \\
\hline
\end{tabular}

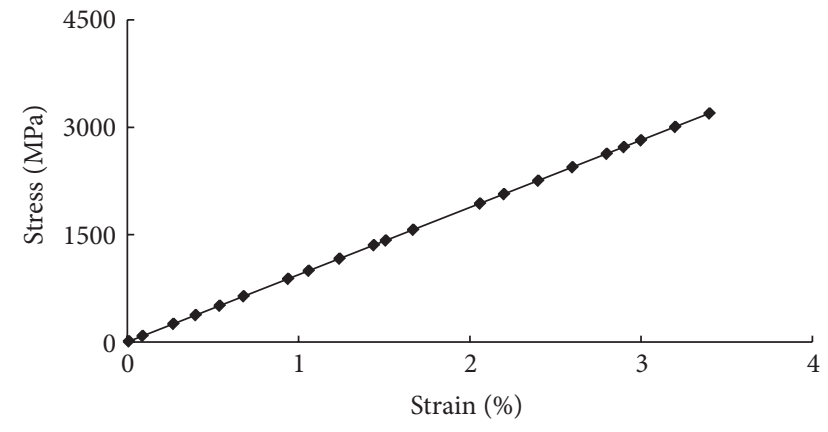

Figure 2: Tensile stress-strain curves of the glass fiber.

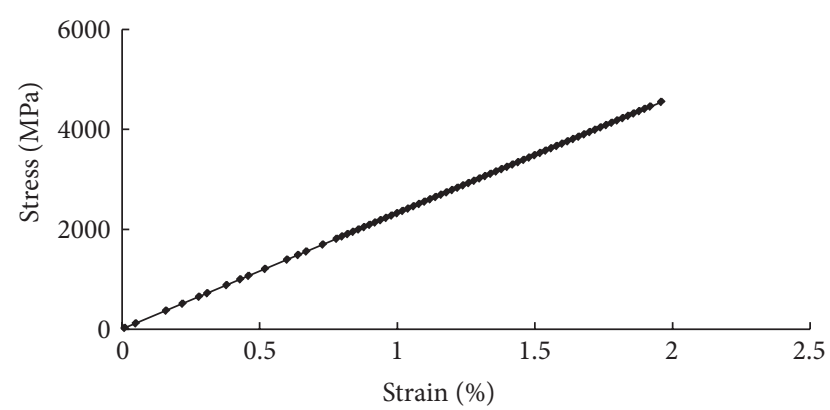

FIGURE 3: Tensile stress-strain curves of the carbon fiber.

at least 5 times greater than the diameter in test standard of GB/T15560. But this long diameter ratio of our test sample is only 2.7. As the shorter sample is sensitive to the effects of the end portion in the testing process, this may be the main reason that the test results are slightly higher than the prediction result. In all, the test results of the static pressure burst test made a good agreement with the prediction result, and it is feasible to verify the overall performance of the cylinder. This test result would be taken as the important reference for the constant stress accelerated life test in the following research.

\section{Accelerated Life Test}

3.1. Experiment Preparation. In the working conditions, the bearing stress of cylinder is about $913.8 \mathrm{Mpa}$, which is equivalent to $45.5 \%$ of the ultimate failure stress. We calculated
TABLE 3: Test result of the static pressure burst test.

\begin{tabular}{lccc}
\hline Number & $\sigma_{b}(\mathrm{MPa})$ & $\varepsilon_{b}(\%)$ & $E(\mathrm{GPa})$ \\
\hline 1 & 2032.4 & 1.88 & 112.5 \\
2 & 2087.6 & 1.87 & 113.3 \\
3 & 2060.9 & 1.94 & 109.9 \\
4 & 2180.9 & 2.02 & 112.3 \\
5 & 2049.0 & 1.85 & 112.2 \\
6 & 2110.5 & 1.93 & 112.3 \\
7 & 1932.4 & 1.84 & 108.2 \\
8 & 2086.7 & 1.91 & 112.6 \\
\hline Mean & 2067.6 & 1.91 & 111.7 \\
Dispersion & $3.44 \%$ & $3.08 \%$ & $1.43 \%$ \\
\hline
\end{tabular}

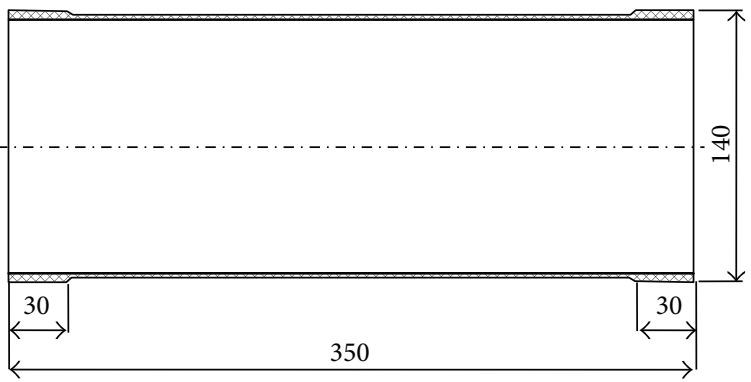

FIGURE 4: Size of the static pressure burst test.

the stress level of the cylinder by the finite element method, and the result shows that the bearing stress of the lining aluminum alloy is $240 \mathrm{MPa}$, the bearing stress of the glass fiber is $1060 \mathrm{Mpa}$, and the bearing of carbon fiber is $1350 \mathrm{MPa}$. The related parameters are shown in Table 1.

The cylinder used in the accelerated life test is the same as that used in the static pressure burst test, as shown in Figure 5. Then, the cylinder is filled with hydraulic oil up to certain pressure and maintains this pressure for a long time so that the cylinder bears a uniform inner pressure in every direction. The test device is placed in one oven where working temperature could be maintained. The pressure can be tested by the pressure sensor. If the pressure of the cylinder drops significantly, the cylinder was considered to be failure and the failure time is automatically recorded. 


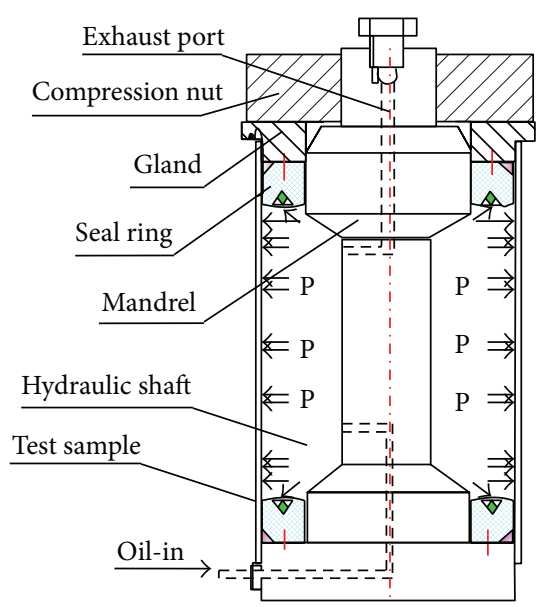

FIGURE 5: Diagram of the reliability test unit of the cylinder.

3.2. The Accelerated Test Plan of the Cylinder. We know the ultimate failure stress is $2067.6 \mathrm{Mpa}$ given by the static pressure burst test and the working stress is $913.8 \mathrm{Mpa}$, which is equivalent to $45.5 \%$ of the ultimate failure stress of the cylinder. The stress-strain curve is close to linear in the scope of the ultimate failure stress of the cylinder.

According to GB 2689.1-81, we divided the cylinders into five groups for constant stress accelerated life test. And we set the minimum stress level to be $1315 \mathrm{MPa}$ (almost $63.6 \%$ of the ultimate failure stress) and the maximum stress level to be $1861 \mathrm{MPa}$ (almost $90 \%$ of the ultimate failure stress). Also, other stress levels were set to be $72.7 \%, 80 \%$, and $85 \%$ of the ultimate failure stress, respectively. According to the test standard of GB 2689.1-81, this test level setting could ensure that the failure mechanism of cylinders was the same. The working temperature of the cylinder will not exceed $40^{\circ} \mathrm{C}$, so the test temperature is controlled at $40 \pm 2^{\circ} \mathrm{C}$.

\section{Results and Discussion}

4.1. The Method on Rupture Life Evaluation of the Cylinder. We assume that there are $n_{j}$ samples prepared for type-I censored test in the $v_{j}(j=1,2, \ldots, s)$ stress level and the censored time is $\xi_{j}^{*}$. There are $q_{j}\left(1 \leq q_{j} \leq n_{j}\right)$ failures and the censored data is $\xi_{j 1} \leq \cdots \leq \xi_{j q_{j}}$.

Hypothesis 1. The product life follows Weibull distribution $W\left(m_{j}, \eta_{j}\right)$ in the $v_{j}$ stress level.

Hypothesis 2. The failure mechanisms of the carbon fiber and the cylinder are the same in each stress level.

Hypothesis 3. The relationship between characteristic life $\eta_{j}$ and stress level $v_{j}$ follows the inverse power law model: $\eta_{j}=$ $A v_{j}^{-c}, j=1,2, \ldots s$. And $A, c$ are parameters that should be estimated.

The linear expression can be obtained by logarithmic transformation on the accelerated model in Hypothesis 3:

$$
\ln \eta_{j}=a+b x_{j} .
$$

Then the logarithmic life follows the extreme value distribution and the relationship between the logarithmic life and the logarithmic stress can be written as

$$
\begin{gathered}
y_{j}=a+b x_{j}+\varepsilon_{j k}, \quad \varepsilon_{j k} \sim E V(0, \sigma), \\
\left(j=1,2, \ldots, s ; k=1,2, \ldots, q_{j}+1\right),
\end{gathered}
$$

where $y_{j}=\ln \eta_{j}, x_{j}=\ln v_{j}, a=\ln A$, and $b=-c$. Parameter $a$ reflects the characteristic of test product; parameter $b$ reflects the acceleration characteristic of the test; $\sigma$ is the scale parameter of the extreme value distribution and the measure parameter for the consistency of the failure mechanism.

In the condition of Hypothesis 1, type-I censored data $y_{j 1} \leq \cdots \leq y_{j q_{j}}$ can be taken as the value of the former $q_{j}$ order statistics $Y_{j 1} \leq \cdots \leq Y_{j q_{j}}$ for the extreme value distribution with size $n_{j} \cdot y_{j\left(q_{j}+1\right)}=y_{j}^{*}$ can be taken as the value of the $q_{j}$ th interval statistics $Y_{j\left(q_{j}+1\right)}$ with the same sample.

From literature $[9,10]$, we can obtain the estimations of $a$, $b$, and $\sigma$ by partial derivative for $Q$

$$
\begin{aligned}
Q=\sum_{j=1}^{s} \sum_{k, l=1}^{q_{j}+1}\left(y_{j k}-a-b x_{j}-\sigma u_{j k}\right) & \\
& \times g_{j k l}\left(y_{j l}-a-b x_{j}-\sigma u_{j l}\right) .
\end{aligned}
$$

The estimations of $a, b$, and $\sigma$ are

$$
\begin{gathered}
\widehat{a}=\bar{y}-\widehat{b} \bar{x}-\widehat{\sigma} \bar{u}, \\
\widehat{b}=\frac{L_{22} L_{1 y}-L_{12} L_{2 y}}{L_{11} L_{22}-L_{12}^{2}}, \\
\widehat{\sigma}=\frac{L_{11} L_{2 y}-L_{12} L_{1 y}}{L_{11} L_{22}-L_{12}^{2}},
\end{gathered}
$$

where

$$
\begin{gathered}
\bar{y}=\frac{1}{n^{*}} \sum_{j=1}^{s} \sum_{k, l=1}^{q_{j}+1} g_{j k l} y_{j k}, \quad \bar{u}=\frac{1}{n^{*}} \sum_{j=1}^{s} \sum_{k, l=1}^{q_{j}+1} g_{j k l} u_{j k}, \\
\bar{x}=\frac{1}{n^{*}} \sum_{j=1}^{s} \sum_{k, l=1}^{q_{j}+1} g_{j k l} x_{j}, \quad n^{*}=\sum_{j=1}^{s} \sum_{k, l=1}^{q_{j}+1} g_{j k l}, \\
L_{1 y}=\sum_{j=1}^{s} \sum_{k, l=1}^{q_{j}+1} g_{j k l}\left(x_{j}-\bar{x}\right)\left(y_{j k}-\bar{y}\right), \\
L_{2 y}=\sum_{j=1}^{s} \sum_{k, l=1}^{q_{j}+1} g_{j k l}\left(u_{j k}-\bar{u}\right)\left(y_{j l}-\bar{y}\right), \\
L_{11}=\sum_{j=1}^{s} \sum_{k, l=1}^{q_{j}+1} g_{j k l}\left(x_{j}-\bar{x}\right)^{2},
\end{gathered}
$$




$$
\begin{aligned}
& L_{12}=\sum_{j=1}^{s} \sum_{k, l=1}^{q_{j}+1} g_{j k l}\left(x_{j}-\bar{x}\right)\left(u_{j k}-\bar{u}\right), \\
& L_{22}=\sum_{j=1}^{s} \sum_{k, l=1}^{q_{j}+1} g_{j k l}\left(u_{j k}-\bar{u}\right)\left(u_{j l}-\bar{u}\right) .
\end{aligned}
$$

And the covariance matrix of $a, b$, and $\sigma$ is

$$
\begin{gathered}
\operatorname{cov}(\widehat{a}, \widehat{b}, \widehat{\sigma})=\sigma^{2} C, \\
C=\left[\begin{array}{ccc}
\sum_{j, k, l} g_{j k l} & \sum_{j, k, l} g_{j k l} x_{j} & \sum_{j, k, l} g_{j k l} \mu_{j k} \\
\sum_{j, k, l} g_{j k l} x_{j} & \sum_{j, k, l} g_{j k l} x_{j}^{2} & \sum_{j, k, l} g_{j k l} x_{j} \mu_{j k} \\
\sum_{j, k, l} g_{j k l} \mu_{j k} & \sum_{j, k, l} g_{j k l} x_{j} \mu_{j k} & \sum_{j, k, l} g_{j k l} \mu_{j k} \mu_{j l}
\end{array}\right]^{-1},
\end{gathered}
$$

where $G=\left(g_{j k l}\right)_{\left(q_{j}+1\right) \times\left(q_{j}+1\right)}=V^{-1}=\left(v_{j k l}\right)_{\left(q_{j}+1\right) \times\left(q_{j}+1\right)}^{-1}, u_{j k}(k=1$, $\left.2, \ldots, q_{j}\right)$ is the mean of the $k$ th order statistic for the standard extreme value distribution with size $n_{j}, v_{j k l}(k, l=$ $\left.1,2, \ldots, q_{j}\right)$ is the covariance of the $k$ th and $l$ th order statistic for the standard extreme value distribution with size $n_{j}, u_{j\left(q_{j}+1\right)}$ is the mean of the $\left(q_{j}+1\right)$ th order statistic for the standard extreme value distribution with size $n_{j}+1$, and $v_{j k\left(q_{j}+1\right)}=v_{j\left(q_{j}+1\right) k}\left(k=1,2, \ldots, q_{j}+1\right)$ is the covariance of the $k$ th and $\left(q_{j}+1\right)$ th order statistic for the standard extreme value distribution with size $n_{j}+1$. These values could be all obtained by formula or table lookup [15].

The reliability rupture life with reliability of $R$ and its upper and lower limits with confidence level $\gamma$ could be calculated as follows:

$$
\begin{gathered}
y_{R}=\widehat{a}+\widehat{b} x+\widehat{\sigma} \ln \ln \frac{1}{R}, \\
y_{R U}=\widehat{a}+\widehat{b} x \\
+\frac{\widehat{\sigma}}{1-u_{\gamma}^{2} c_{33}}\left[\ln \ln \frac{1}{R}+u_{\gamma}^{2}\left(c_{13}+c_{23} x\right)+u_{\gamma} \sqrt{\omega_{R}}\right], \\
y_{R L}=\widehat{a}+\widehat{b} x \\
+\frac{\widehat{\sigma}}{1-u_{\gamma}^{2} c_{33}}\left[\ln \ln \frac{1}{R}+u_{\gamma}^{2}\left(c_{13}+c_{23} x\right)-u_{\gamma} \sqrt{\omega_{R}}\right],
\end{gathered}
$$

where $c_{i j}$ are elements of the matrix $C, \omega_{R}=\omega+c_{33}(\ln \ln (1 / R))^{2}$ $+2\left(c_{13}+c_{23} x\right) \ln \ln (1 / R)$, and $\omega=u_{\gamma}^{2}\left(c_{13}+c_{23} x\right)^{2}+\left(1-c_{33} u_{\gamma}^{2}\right)\left(c_{11}+\right.$ $\left.2 c_{12} x+c_{22} x^{2}\right)$.

The Failure Mechanism Consistency Analysis. This analysis focused on the consistency of failure mechanism and accelerated model parameter between the carbon fiber and cylinder in the accelerated life test. Denote the model parameters of the carbon fiber and the cylinder as $a_{1}, b_{1}$, and $\sigma_{1}$ and $a_{2}, b_{2}$, and $\sigma_{2}$, respectively. We assume the following.
(1) The distribution parameters $\sigma_{1}, \sigma_{2}$ are two independent normal populations. If the failure mechanism of the carbon fiber is the same with the cylinder, the mean and variance of the two normal populations are the same $[16,17]$.

(2) Parameters of $a_{1}$ and $a_{2}$ reflect the life characteristic of carbon fiber and cylinder, and then $a_{1}$ and $a_{2}$ have no relation.

(3) The model parameters $b_{1}, b_{2}$ are two independent normal populations. If the acceleration of the carbon fiber is the same with the cylinder, the mean and variance of the two normal populations are the same.

Based on the above assumptions, $\left(\widehat{b}_{1}, \widehat{\sigma}_{1}\right)$ and $\left(\widehat{b}_{2}, \widehat{\sigma}_{2}\right)$ can be taken as two bivariate normal populations. We can judge the consistency of the mean vector and covariance matrix of the two bivariate normal populations by hypothesis test.

(1) The Consistency Judgment of the Mean Vector. The two independent normal populations are denoted by $\left(\widehat{b}_{1}, \widehat{\sigma}_{1}\right) \sim$ $N_{2}\left(\mu_{1}, \Sigma_{1}\right)$ and $\left(\widehat{b}_{2}, \widehat{\sigma}_{2}\right) \sim N_{2}\left(\mu_{2}, \Sigma_{2}\right)$. We sample $n, m>$ 2 specimens from them, respectively, and denote the mean vectors by $\bar{X}, \bar{Y}$, respectively, and the variance matrix by $S_{i}(i=1,2)$. The hypothesis is

$$
H_{0}: \mu_{1}=\mu_{2} \quad H_{1}: \mu_{1} \neq \mu_{2} .
$$

When $\Sigma_{1}=\Sigma_{2}$ and they were unknown, the test statistic

$$
T^{2}=\frac{n m}{n+m}(\bar{X}-\bar{Y})^{T} S^{-1}(\bar{X}-\bar{Y}),
$$

where

$$
S=\frac{(n-1) S_{1}+(m-1) S_{2}}{n+m-2} .
$$

And $F=(((n+m-2)-1) / 2(n+m-2)) T^{2} \sim F(2, n+m-3)$.

Then the rejection region with the significance level $\alpha$ is

$$
\left\{F>F_{1-\alpha}(2, n+m-3)\right\} .
$$

(2) The Consistency Judgment of the Variance Matrix. The hypothesis is

$$
H_{0}: \Sigma_{1}=\Sigma_{2} \quad H_{1}: \Sigma_{1} \neq \Sigma_{2} .
$$

The amendatory likelihood ratio statistic is

$$
\lambda^{*}=\frac{(n+m-2)^{(n+m-2)}\left|S_{1}\right|^{(n-1) / 2}\left|S_{2}\right|^{(m-1) / 2}}{(n-1)^{(n-1) / 2}(m-1)^{(m-1) / 2}\left|S_{1}+S_{2}\right|^{(n+m-2) / 2}} .
$$

Then,

$$
-2 \ln \lambda^{*} \sim \chi^{2}\left(\frac{f}{1-d}\right),
$$


where

$$
\begin{gathered}
f=\frac{1}{2} p(p+1)(k-1), \\
d=\frac{2 p^{2}+3 p-1}{6(p+1)(k-1)}\left(\frac{1}{n-1}+\frac{1}{m-1}-\frac{1}{n+m-k}\right)
\end{gathered}
$$

and $k=p=2$.

Then the rejection region with the significance level $\alpha$ is

$$
\left\{\chi^{2}<\chi_{\alpha}^{2}\left(\frac{f}{1-d}\right)\right\} .
$$

The information fusion method

Based on the result of the integral best linear unbiased estimation and the consistency analysis of failure mechanism, we proposed an information fusion method for the covariance matrixes of the T700 carbon fiber and the cylinder. The fusion process can be accomplished by the two steps: firstly, fusing the information only related to parameters $b$ and $\sigma$ in the covariance matrix and then, secondly, to further improve the evaluation accuracy of the covariance matrix for model parameter of the cylinder, fusing the other information related to $b$ and $\sigma$.

Denote the point estimations of T700 carbon fiber and the cylinder by $\widehat{a}_{1}, \widehat{b}_{1}$, and $\widehat{\sigma}_{1}$ and $\widehat{a}_{2}, \widehat{b}_{2}$, and $\widehat{\sigma}_{2}$, respectively, and their covariance matrix is

$$
\begin{aligned}
& \operatorname{cov}\left(\widehat{a}_{i}, \widehat{b}_{i}, \widehat{\sigma}_{i}\right)=\left[\begin{array}{ccc}
\operatorname{var}\left(\widehat{a}_{i}\right) & \operatorname{cov}\left(\widehat{a}_{i}, \widehat{b}_{i}\right) & \operatorname{cov}\left(\widehat{a}_{i}, \widehat{\sigma}_{i}\right) \\
\operatorname{cov}\left(\widehat{a}_{i}, \widehat{b}_{i}\right) & \operatorname{var}\left(\widehat{b}_{i}\right) & \operatorname{cov}\left(\hat{b}_{i}, \widehat{\sigma}_{i}\right) \\
\operatorname{cov}\left(\widehat{a}_{i}, \widehat{\sigma}_{i}\right) & \operatorname{cov}\left(\widehat{b}_{i}, \widehat{\sigma}_{i}\right) & \operatorname{var}\left(\widehat{\sigma}_{i}\right)
\end{array}\right], \\
& (i=1,2) \text {. }
\end{aligned}
$$

Denote the covariance matrix of the bivariate normal distribution of $b_{1}, \sigma_{1}$ and $b_{2}, \sigma_{2}$, respectively, by

$$
\widehat{V}_{i}=\left[\begin{array}{cc}
\operatorname{var}\left(\widehat{b}_{i}\right) & \operatorname{cov}\left(\widehat{b}_{i}, \widehat{\sigma}_{i}\right) \\
\operatorname{cov}\left(\widehat{b}_{i}, \widehat{\sigma}_{i}\right) & \operatorname{var}\left(\widehat{\sigma}_{i}\right)
\end{array}\right], \quad(i=1,2) .
$$

The elements of the above matrixes are one part of (19).

The first step is fusing the information only related to parameters $b$ and $\sigma$ in the covariance matrix.

If the covariance matrixes $V_{1}$ and $V_{2}$ are certified to be the same by the consistency analysis of the bivariate normal distribution $\left(b_{1}, \sigma_{1}\right)$ and $\left(b_{2}, \sigma_{2}\right)$, the unbiased estimation of the covariance matrix of the bivariate normal distribution can be obtained by the following equation:

$$
\widehat{V}=\frac{\left(n_{1}-1\right) \widehat{V}_{1}+\left(n_{2}-1\right) \widehat{V}_{2}}{n_{1}+n_{2}-2} .
$$

Compared to the small ratio of failure and high censored time of the cylinder life test, the data and failures of the carbon fiber are much greater and thus the evaluation result is more accurate. Therefore, when the matrix $\widehat{V}_{2}$ is replaced by $\widehat{V}$ with fusing the information of parameters $b$ and $\sigma$ according to the above method, the prediction result of the cylinder is more accurate. The covariance matrix of $b$ and $\sigma$ based on fusion could be written as

$$
\widehat{V}=\left[\begin{array}{cc}
\operatorname{var}\left(\widehat{b}_{12}\right) & \operatorname{cov}\left(\widehat{b}_{12}, \widehat{\sigma}_{12}\right) \\
\operatorname{cov}\left(\widehat{b}_{12}, \widehat{\sigma}_{12}\right) & \operatorname{var}\left(\widehat{\sigma}_{12}\right)
\end{array}\right] .
$$

The covariance matrix $\operatorname{cov}\left(\widehat{a}_{2}, \widehat{b}_{2}, \widehat{\sigma}_{2}\right)$ for the cylinder with bottom right four elements replaced by $\widehat{V}$ could be written as

$$
\begin{aligned}
& \operatorname{cov}\left(\widehat{a}_{2}, \widehat{b}_{2}, \widehat{\sigma}_{2}\right)^{\prime} \\
& \quad=\left[\begin{array}{ccc}
\operatorname{var}\left(\widehat{a}_{2}\right) & \operatorname{cov}\left(\widehat{a}_{2}, \widehat{b}_{2}\right) & \operatorname{cov}\left(\widehat{a}_{2}, \widehat{\sigma}_{2}\right) \\
\operatorname{cov}\left(\widehat{a}_{2}, \widehat{b}_{2}\right) & \operatorname{var}\left(\widehat{b}_{12}\right) & \operatorname{cov}\left(\widehat{b}_{12}, \widehat{\sigma}_{12}\right) \\
\operatorname{cov}\left(\widehat{a}_{2}, \widehat{\sigma}_{2}\right) & \operatorname{cov}\left(\widehat{b}_{12}, \widehat{\sigma}_{12}\right) & \operatorname{var}\left(\widehat{\sigma}_{12}\right)
\end{array}\right] .
\end{aligned}
$$

The second step is fusing the other elements related to $b, \sigma$ of the covariance matrix.

Based on the same correlation coefficient between the parameters, the value of $\operatorname{cov}\left(\widehat{a}_{2}, \widehat{b}_{12}\right), \operatorname{cov}\left(\widehat{a}_{2}, \widehat{\sigma}_{12}\right)$ with fusing the information of the T700 carbon fiber can be calculated as

$$
\begin{aligned}
& \operatorname{cov}\left(\widehat{a}_{2}, \widehat{b}_{12}\right)=\sqrt{\frac{\operatorname{var}\left(\widehat{b}_{12}\right)}{\operatorname{var}\left(\widehat{b}_{2}\right)}} \cdot \operatorname{cov}\left(\widehat{a}_{2}, \widehat{b}_{2}\right), \\
& \operatorname{cov}\left(\widehat{a}_{2}, \widehat{\sigma}_{12}\right)=\sqrt{\frac{\operatorname{var}\left(\widehat{\sigma}_{12}\right)}{\operatorname{var}\left(\widehat{\sigma}_{2}\right)}} \cdot \operatorname{cov}\left(\widehat{a}_{2}, \widehat{\sigma}_{2}\right) .
\end{aligned}
$$

According to the above two steps, the covariance matrix $\operatorname{cov}\left(\widehat{a}_{2}, \widehat{b}_{12}, \widehat{\sigma}_{12}\right)$ can be written as

$$
\begin{aligned}
& \operatorname{cov}\left(\widehat{a}_{2}, \widehat{b}_{12}, \widehat{\sigma}_{12}\right) \\
& =\left[\begin{array}{ccc}
\operatorname{var}\left(\widehat{a}_{2}\right) & \operatorname{cov}\left(\widehat{a}_{2}, \widehat{b}_{12}\right) & \operatorname{cov}\left(\widehat{a}_{2}, \widehat{\sigma}_{12}\right) \\
\operatorname{cov}\left(\widehat{a}_{2}, \widehat{b}_{12}\right) & \operatorname{var}\left(\widehat{b}_{12}\right) & \operatorname{cov}\left(\widehat{b}_{12}, \widehat{\sigma}_{12}\right) \\
\operatorname{cov}\left(\widehat{a}_{2}, \widehat{\sigma}_{12}\right) & \operatorname{cov}\left(\widehat{b}_{12}, \widehat{\sigma}_{12}\right) & \operatorname{var}\left(\widehat{\sigma}_{12}\right)
\end{array}\right] .
\end{aligned}
$$

Comparing with (25) and (19) $(i=2)$, it can be found that the elements of parameter covariance matrix of the cylinder have changed in addition to the variance vâr $\left(\widehat{a}_{2}\right)$ by fusing the carbon fiber test information, thus making the evaluation result more reasonable. It should be noted that the parameter covariance matrix can be obtained by the integrated best linear unbiased estimation as follows:

$$
\operatorname{cov}(\widehat{a}, \widehat{b}, \widehat{\sigma})=\sigma^{2} C .
$$

In the calculation of the upper and lower limits for the reliability rupture life, the matrix $C$ would be used. Therefore, in fusion process of the covariance matrix with T700 carbon fiber information and cylinder information, we can introduce the matrix $C$ directly in the above method to make the calculation easier.

4.2. Examples. To verify the design level of the rupture life of a certain type of cylinder, a unit made accelerated life tests for T700 carbon fiber composite material and the cylinder 
TABLE 4: Accelerated life test information for T700 carbon fiber and the cylinder.

\begin{tabular}{lccccc}
\hline Subjects & \multicolumn{5}{c}{ Stress level } \\
\hline $\begin{array}{l}\text { T700 carbon fiber }(N) \\
\begin{array}{l}\text { Cylinder (the percentage of limit } \\
\text { load \%) }\end{array}\end{array}$ & 63.6 & 72.7 & 80 & 85 & 90 \\
\hline
\end{tabular}

structure, respectively. The test temperature is $40^{\circ} \mathrm{C}$, and the test load conditions are shown in Table 4.

The diagram of rupture life data for T700 carbon fiber and the cylinder structure from the test is as in Figures 6 and 7.

(1) The Result of the Integral Best Unbiased Estimation. The results of model parameters and the matrix $C$ obtained by the integrated best linear unbiased estimation are shown in Table 5.

(2) The Test of Mean Vector and Covariance Matrix Equal to $(\widehat{b}, \widehat{\sigma})$ of the Carbon Fiber and the Cylinder. We consider parameters $(\widehat{b}, \widehat{\sigma})$ as a bivariate normal population and test whether the mean vectors and covariance matrixes of $(\widehat{b}, \widehat{\sigma})$ for the carbon fiber and the cylinder are equal or not, and the results are shown in Table 6.

From Table 6, the observed values of the test statistic are

$$
\begin{gathered}
F=8.09725<F_{0.975}(2,5)=8.43, \\
\chi^{2}=1.9468>\chi_{0.025}^{2}(5) .
\end{gathered}
$$

Then, we can receive the null hypothesis. The mean vectors and the covariance matrixes of $(\widehat{b}, \widehat{\sigma})$ for the carbon fiber and the cylinder are equal.

(3) The Information Fusion of the T700 Carbon Fiber and the Cylinder. By using the method in this paper, the matrix information of carbon fiber in Table 5 can be fused into the cylinder and the upper and lower limits of the reliability rupture life at confidence level $\gamma=0.95$ can be calculated. The comparison results are shown in Table 7.

From Table 7, when the reliability is 0.9 , the evaluation accuracy of the reliability life of the cylinder is increased by $35 \%$.

The curves of upper and lower limits of the logarithmic reliability rupture life $y_{R}$ changed with the reliability $R$ are shown in Figure 7.

Figure 8 shows that the upper and lower limits of the reliability rupture life will be more accurate with the reliability changed after fusion of the information of T700 carbon fiber and the interval length is much shorter.

\section{Conclusion}

(1) Since the carbon fiber bears the main load at work, the acceleration and failure mechanism of T700 carbon fiber and the cylinder are the same. And it can be proved by the structure analysis and the statistic test of the test data.

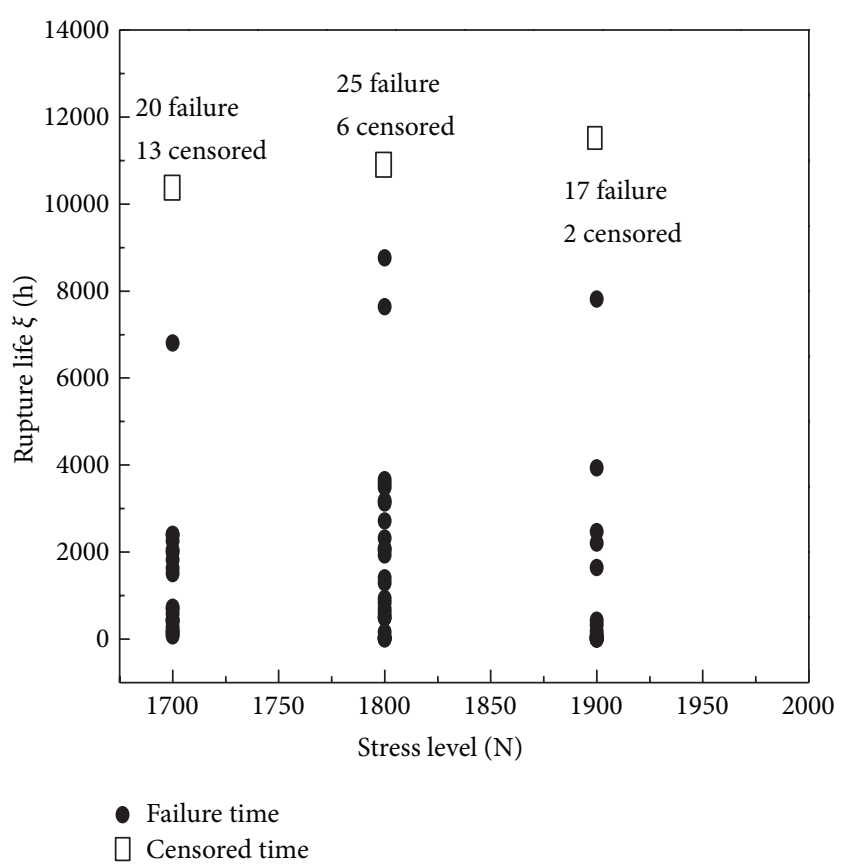

Figure 6: Rupture life of T700 carbon fiber.

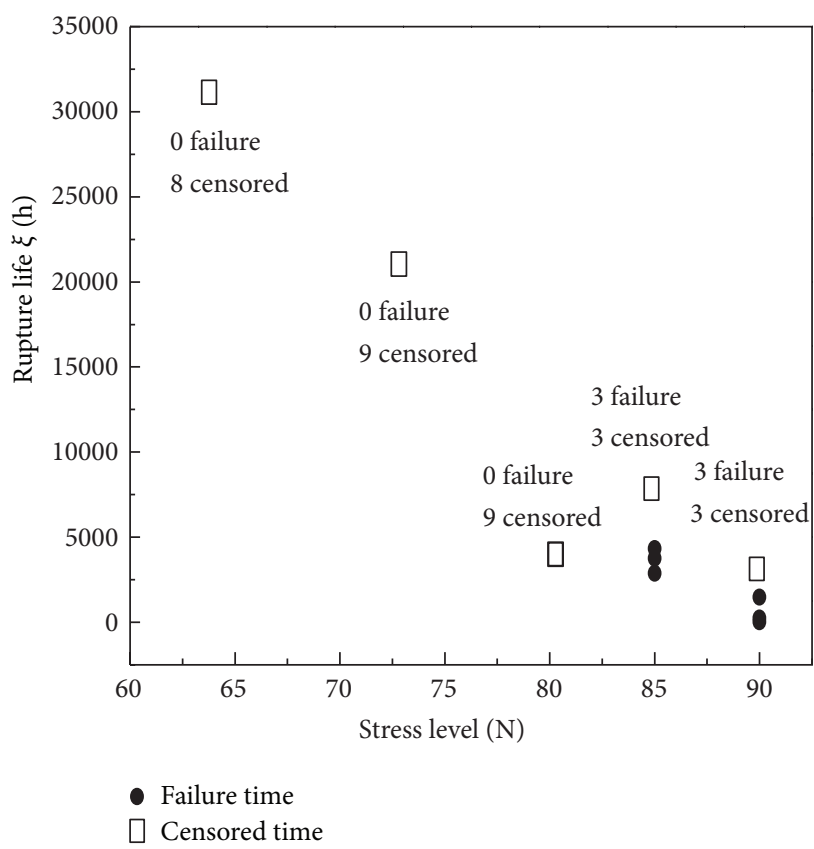

Figure 7: Ruptures life of the cylinder.

(2) When the acceleration and failure mechanism are the same, the evaluation accuracy of the reliability life for the cylinder can be improved by fusion of the information of the carbon fiber.

(3) The method in this paper is based on Weibull distribution and the inverse power law model for structured products. It can be applied to other locationscale family distribution and acceleration models. 
TABLE 5: Contrast result between T700 carbon fiber and the cylinder with the integrated best linear unbiased estimation.

\begin{tabular}{|c|c|c|c|c|c|}
\hline Result & \multicolumn{2}{|c|}{ T700 carbon fiber } & \multicolumn{3}{|c|}{ Cylinder } \\
\hline$(\widehat{a}, \widehat{b}, \widehat{\sigma})$ & \multicolumn{2}{|c|}{$(157.177,-19.872,2.32)$} & \multicolumn{3}{|c|}{$(6.39,-18.41,1.20)$} \\
\hline \multirow{3}{*}{ C } & 495.0 & $-66.08 \quad 0.46$ & 0.53 & 2.70 & -0.06 \\
\hline & -66.08 & $8.82-0.06$ & 2.70 & 21.49 & -1.05 \\
\hline & L 0.46 & $-0.06 \quad 0.012$ & -0.06 & -1.05 & 0.13 \\
\hline
\end{tabular}

TABLE 6: The test results of mean vector and covariance matrix for a bivariate normal population $(\widehat{b}, \widehat{\sigma})$ of carbon fiber and cylinder.

\begin{tabular}{lccc}
\hline Null hypothesis & Rejection region & Observation value & Critical value $\alpha=0.025$ \\
\hline$\mu_{1}=\mu_{2}$ & $\left\{F>F_{1-\alpha}(2, n+m-3)\right\}$ & 8.09725 & 8.43 \\
\hline$\Sigma_{1}=\Sigma_{2}$ & $\left\{\chi^{2}<\chi_{\alpha}^{2}(f /(1-d))\right\}$ & 1.9468 & 0.831 \\
\hline
\end{tabular}

TABLE 7: Contrast result of cylinder after fusion information of T700 carbon fiber.

\begin{tabular}{|c|c|c|c|}
\hline Result & The original data & \multicolumn{2}{|c|}{ Fusion of the information of T700 } \\
\hline \multirow{3}{*}{ C } & $\begin{array}{lll}0.53 & 2.70 & -0.06\end{array}$ & $0.53 \quad 2.08$ & -0.037 \\
\hline & $2.70 \quad 21.49-1.05$ & $2.08 \quad 12.76$ & -0.37 \\
\hline & {$\left[\begin{array}{lll}-0.06 & -1.05 & 0.13\end{array}\right]$} & $\begin{array}{ll}-0.037 & -0.37 \\
\end{array}$ & 0.049 \\
\hline The interval estimation of $y_{R}$ & {$[13.79,27.35]$} & \multicolumn{2}{|c|}{$[14.29,23.15]$} \\
\hline Interval length & 13.56 & \multicolumn{2}{|l|}{8.86} \\
\hline
\end{tabular}

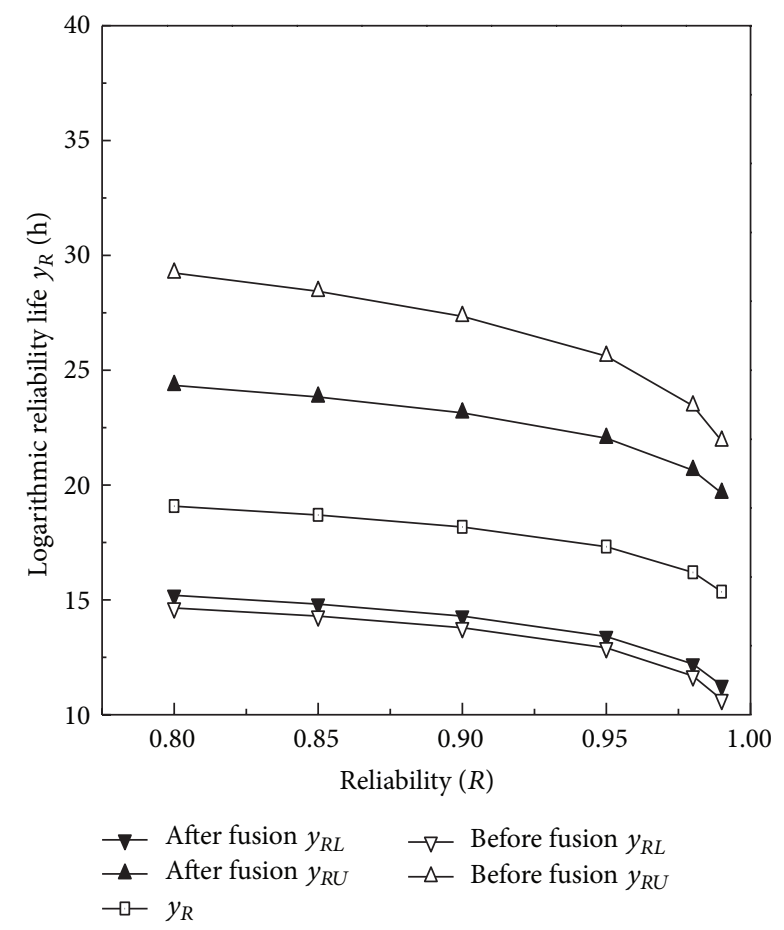

FIGURE 8: Change curves for $y_{R}$ and upper and lower limits.

\section{Conflict of Interests}

The authors declare that there is no conflict of interests regarding the publication of this paper.

\section{Acknowledgments}

The work described in this paper was supported by the National Natural Science Foundation of China (Grants nos. 61104133 and 61473014). The authors fully appreciate the financial support. The authors would like to thank the reviewers and the editor for many suggestions that helped improve this paper.

\section{References}

[1] D. F. Li, H. J. Wang, and F. He, "Structure and properties of T300 and T700 carbon fiber," New Carbon Materials, vol. 22, no. 1, pp. 59-64, 2007.

[2] G. T. Zhang, W. G. Chen, and B. Yang, "Testing research on mechanical properties of T700 carbon fiber/epoxy composites," Fiber Composites, vol. 2, pp. 49-52, 2009.

[3] Y. X. Zhou, M. A. Baseer, H. Mahfuz, and S. Jeelani, "Statistical analysis on the fatigue strength distribution of T700 carbon fiber," Composites Science and Technology, vol. 66, no. 13, pp. 2100-2106, 2006.

[4] Z. G. Yu, S. C. Yang, and B. F. Song, "Comparison of wet and hot aging resistance of T700 and T300 carbon fiber strengthened epoxy resin composites," Materials for Mechanical Engineering, vol. 33, no. 6, pp. 48-51, 2009.

[5] X. Gu and X. Xu, "Numerical simulation of damage in fiber reinforced composite laminates under high velocity impact," Acta Materiae Compositae Sinica, vol. 29, no. 1, pp. 150-161, 2012.

[6] J. Liu, Y. X. Bai, Y. L. Tian, X. Y. Huang, C. H. Wang, and J. Y. Liang, "Effect of the process of electrochemical modification on the surface structure and properties of PAN-based carbon firbers," Acta Materiae Compositae Sinica, vol. 29, no. 2, pp. 16$25,2012$. 
[7] W. Yurkosky, R. E. Schafter, and J. M. Finkerlstein, "Accelerated testing technology," Tech. Rep. NO.RADC-TR-67-420, 1-2, 1967.

[8] J. S. Zhao, G. M. Zhuang, and Z. G. Wang, "The introduction of the maximum likelihood estimation method," Journal of Changchun University of Science and Technology, vol. 5, no. 6, pp. 53-54, 2010.

[9] H.-M. Fu and X.-R. Yue, "Regression analysis method for typeIcensored data," Journal of Aerospace Power, vol. 25, no. 1, pp. 142-147, 2010.

[10] X. Ma, T. Wang, J. Wang, and Z. Liu, "Method on accelerated rupture life evaluation for composite material based on type-II censored data," Advanced Materials Research, vol. 284-286, pp. 439-443, 2011.

[11] A. J. Watkins, "Review: Likelihood method for fitting Weibull log-linear models to accelerated life-test data," IEEE Transactions on Reliability, vol. 43, no. 3, pp. 361-365, 1994.

[12] G. Q. Jiao and P. R. Jia, Mechanics of Composites, Northwestern Polytechnical University Press, Xian, China, 2008.

[13] L. F. Gui and Y. T. Cao, Handbook of Mechanical Engineering Materials Testing, Liaoning Science and Technology Press, Liaoning, China, 2001.

[14] J. H. Sun and Y. H. Wang, "Standard test method for short-time hydraulic failure and resistance to constant internal pressure of the plastics pipes for the transport of fluids," GB/T15560, China, 1995.

[15] P. S. B. Chan, "Order statistics from extreme value distribution: I-table of mean, variances and covariances," Communications in Statistics-Simulation and Computation, vol. 21, no. 4, pp. 11991217, 1992.

[16] Y. Q. Zhou, Z. X. Weng, and X. T. Ye, "Study on accelerated factor and condition for constant failure mechanism (I) - the case for lifetime is a random variable," Journal of Systems Engineering and Electronics, vol. 1, pp. 55-67, 1996.

[17] Z. L. Sun, "A condition for constant failure mechanism," Electronic Product Reliability and Environmental Testing, vol. 26, no. 4, pp. 6-8, 2008. 


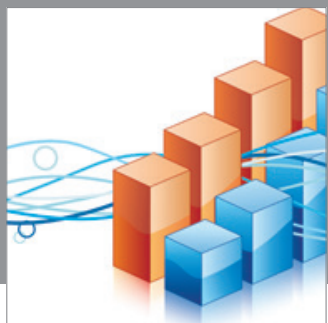

Advances in

Operations Research

mansans

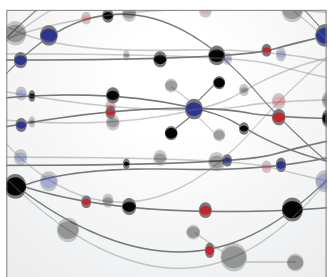

The Scientific World Journal
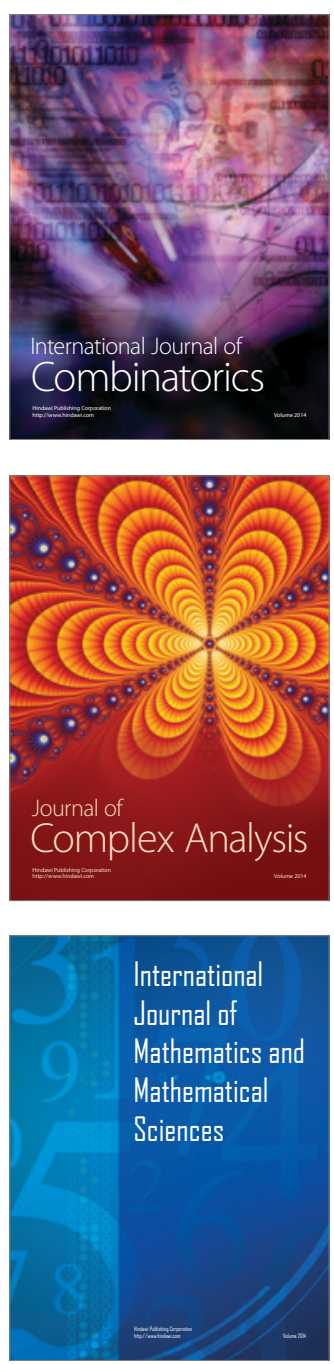
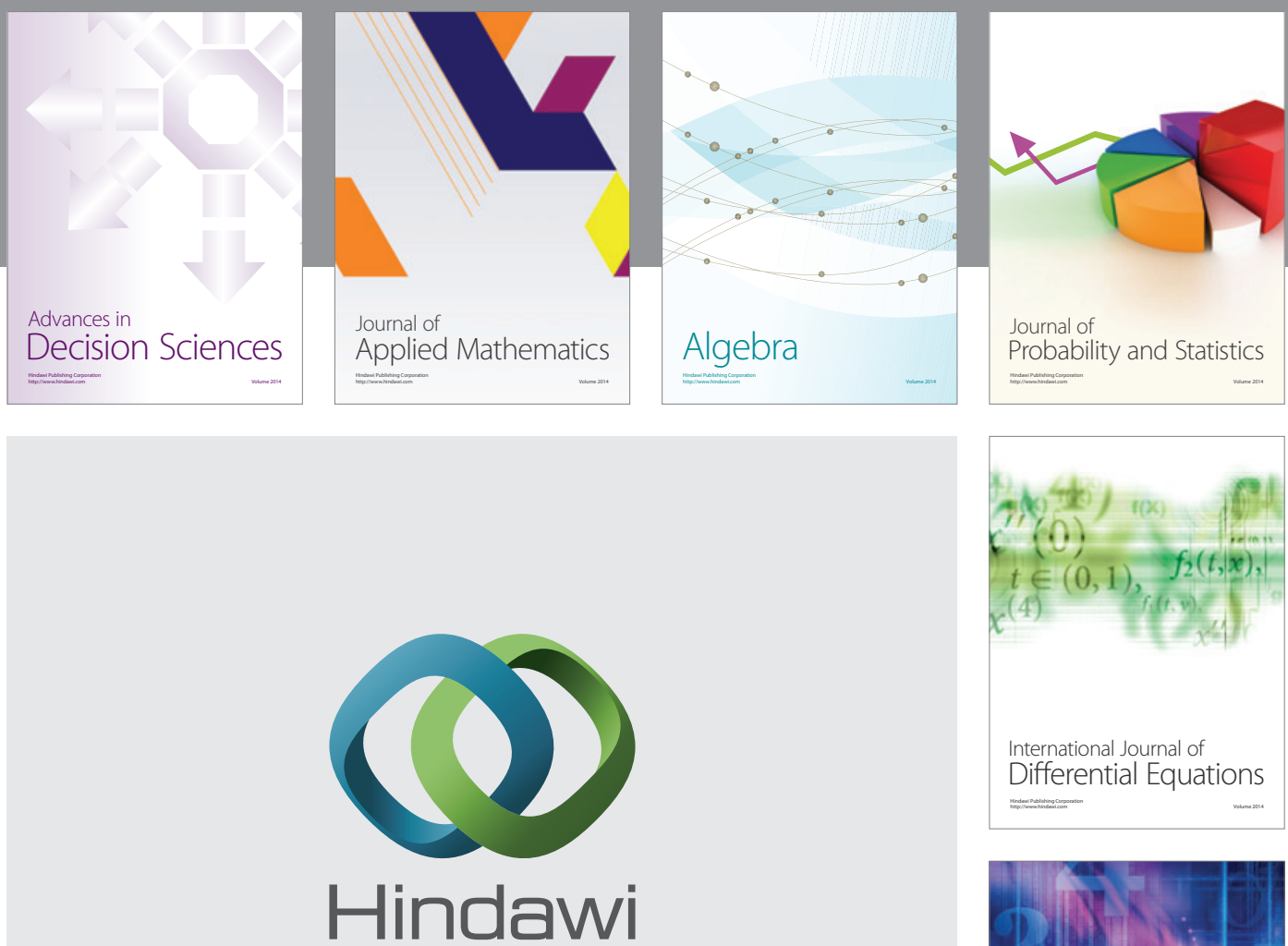

Submit your manuscripts at http://www.hindawi.com
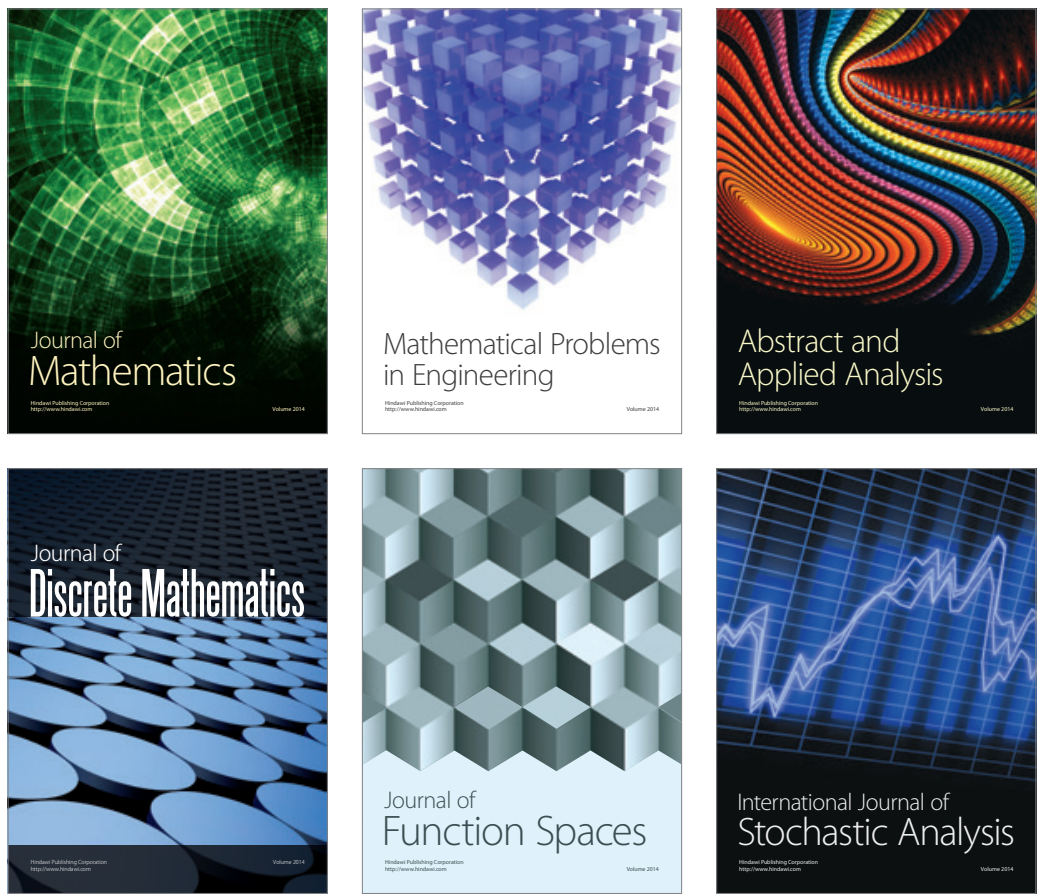

Journal of

Function Spaces

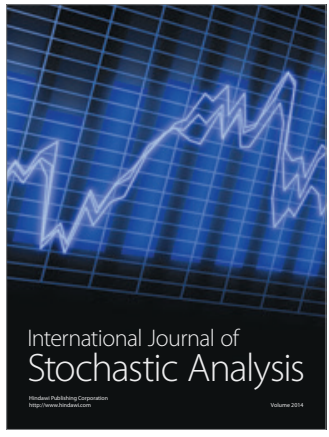

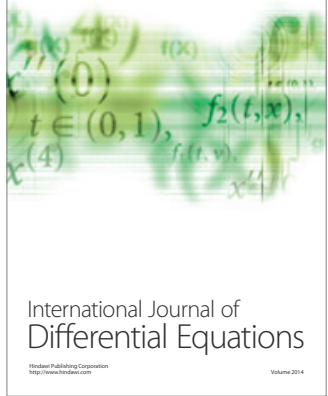
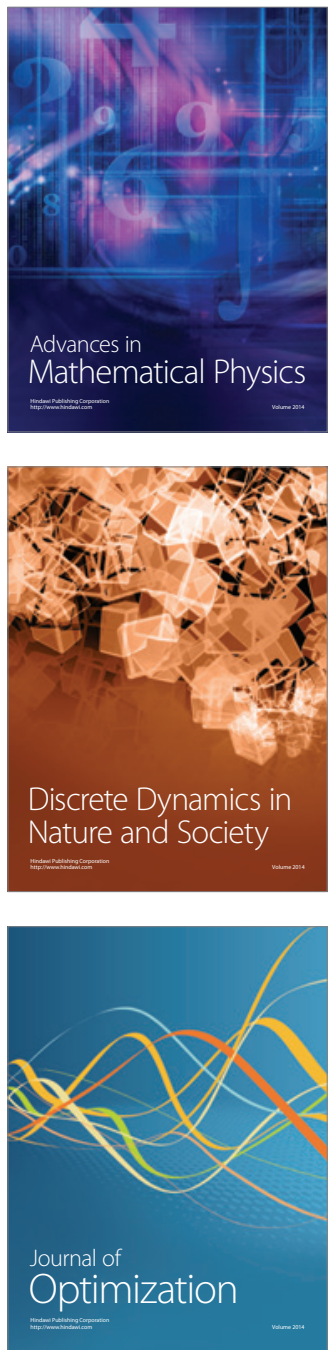